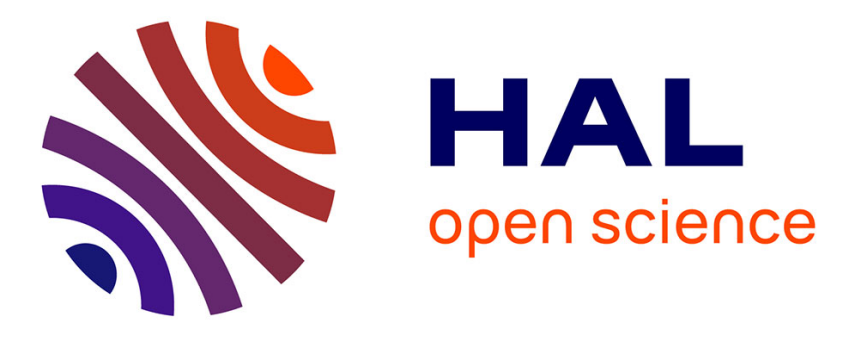

\title{
Mobility and height detection of particle labels in an optical evanescent wave biosensor with single-label resolution
}

Kim van Ommering, Philip A Somers, Marjo Koets, Jean J H B Schleipen, Leo J van Ijzendoorn, Menno W J Prins

\section{To cite this version:}

Kim van Ommering, Philip A Somers, Marjo Koets, Jean J H B Schleipen, Leo J van Ijzendoorn, et al.. Mobility and height detection of particle labels in an optical evanescent wave biosensor with singlelabel resolution. Journal of Physics D: Applied Physics, 2010, 43 (15), pp.155501. 10.1088/0022$3727 / 43 / 15 / 155501$. hal-00569578

\section{HAL Id: hal-00569578 \\ https://hal.science/hal-00569578}

Submitted on 25 Feb 2011

HAL is a multi-disciplinary open access archive for the deposit and dissemination of scientific research documents, whether they are published or not. The documents may come from teaching and research institutions in France or abroad, or from public or private research centers.
L'archive ouverte pluridisciplinaire HAL, est destinée au dépôt et à la diffusion de documents scientifiques de niveau recherche, publiés ou non, émanant des établissements d'enseignement et de recherche français ou étrangers, des laboratoires publics ou privés. 


\title{
Mobility and height detection of particle labels in an optical evanescent wave biosensor with single-label resolution
}

\author{
Kim van Ommering ${ }^{1,2}$, Philip A Somers ${ }^{2}$, Marjo Koets ${ }^{1}$, Jean J H B Schleipen ${ }^{1}$, Leo J van IJzendoorn ${ }^{2}$ and \\ Menno W J Prins ${ }^{1,2}$ \\ ${ }^{1}$ Philips Research Laboratories, 5656 AE Eindhoven, The Netherlands \\ ${ }^{2}$ Department of Applied Physics, Eindhoven University of Technology, 5600 MB Eindhoven, The \\ Netherlands \\ E-mail: kim.van.ommering@philips.com and menno.prins@philips.com
}

\begin{abstract}
Particle labels are used in biosensors to detect the presence and concentration of analyte molecules. In this paper we demonstrate an optical technique to measure the mobility and height of bound particle labels on a biosensor surface with single-label resolution. The technique is based on the detection of the particle-induced light scattering in an optical evanescent field. We show that the thermal particle motion in the evanescent optical field leads to intensity fluctuations that can accurately be detected. The technique is demonstrated using $290 \mathrm{bp}$ (99 nm) DNA as an analyte, and using polystyrene particles and magnetic particles with diameters between $500 \mathrm{~nm}$ and $1000 \mathrm{~nm}$ as labels. The particle intensity histograms show that quantitative height measurements are obtained for particles with uniform optical properties, and the intensity versus position plots reflect the analyte-antibody orientation and the analyte flexibility. The novel optical detection technique will lead to biosensors with very high sensitivity and specificity.
\end{abstract}

\section{PACS}

75.50.Tt

78.67.Bf

87.14.gk

87.64.M-

87.80.Nj

87.85.fk

Submitted to

Journal of Physics D: Applied Physics 


\section{Introduction}

Particle labels are commonly used in biosensors because an analyte coupled to a particle label is more easily detected than just an analyte molecule. Particle labels are often made of polymer material, can be suitably activated with biological molecules, and can be detected by optical means (Seydack 2005, Wild, 2006). For maximum sensitivity and specificity, the ultimate goal in biosensing is to achieve single-molecule resolution and to measure detailed properties of every bound label and every captured molecule. The detection of individual particle labels in biosensor assays has been demonstrated (Mulvaney et al 2009). However, the potential to simultaneously measure detailed dynamic properties of both labels and captured molecules is still to be explored.

We propose to exploit particle-induced light scattering in an optical evanescent field to extract detailed information on binding in a biosensor system. The use of an evanescent field to measure the height of particles near a surface was introduced by Prieve et al $(1987,1999)$ and has mainly been used to study the kinetics and scattering properties of unbound particles near a surface (Sholl et al 2000, Banerjee and Kihm 2005, Blickle et al 2005, Oetama and Walz 2005). Blumberg et al (2005) have shown that an evanescent field can be used to measure the three-dimensional mobility of fluorescent polystyrene particles in tethered particle motion experiments, with tether lengths of 0.4-0.5 $\mu \mathrm{m}$. By determining mean square displacements, time constants and symmetry criteria, they were able to distinguish properly formed tethers from malformed ones.

In this work, we bring the technique of mobility detection using an evanescent field to the field of biosensing. We design a compact biosensor set-up suitable for rapid assays and for the detection of the dynamic properties of bound assay labels. We focus on the use of assay labels with diameters between 500 and $1000 \mathrm{~nm}$ dedicated for biosensing. In addition to polystyrene particles, we pay particular attention to the use of superparamagnetic particles, because such particles can significantly improve assay times and are increasingly being used as biosensor labels (Graham et al 2004, Osterfeld et al 2008, Bruls et al 2009). We determine the requirements of the optical detection system in order to be able to measure the mobility of individual biologically bound particles with nanometre resolution. We experimentally determine the magnitude of intensity fluctuations induced by the three-dimensional Brownian motion of bound particles. We quantify the precision of positional detection of the bound particle labels by using an analyte molecule with defined length, and we discuss how intensity and in-plane position data reflect the analyte-antibody orientation and the analyte flexibility.

\section{Theoretical estimations}

In the design of a biosensor technique suitable for nanometre height detection by intensity measurements we took into consideration two important aspects, namely the accuracy and stability of the evanescent field depth as well as the time resolution of the measurement. The scattered light intensity $I$ of a particle in an evanescent field decays exponentially as function of the height $h$ (Prieve et al 1987):

$$
I(h)=I_{0} e^{-h / d_{p}},
$$

where $I_{0}$ is the intensity of the particle in contact with the surface and $d_{p}$ is the penetration depth of the evanescent field depth given by:

$$
d_{p}=\left[4 \pi / \lambda \sqrt{\left(n_{1} \sin \theta\right)^{2}-n_{2}^{2}}\right]^{-1},
$$

with $\lambda$ the wavelength of the light, $n_{1}$ the refractive index of the first medium (i.e. the sensor cartridge), $n_{2}$ the refractive index of the second medium (i.e. the fluid), and $\theta$ the angle of incidence of the exciting optical beam. Typical values in our experiment are a wavelength $\lambda=650 \mathrm{~nm}$, refractive indices $n_{1}=1.5, n_{2}=1.3$ and an angle of incidence $\theta=70^{\circ}$, leading to an evanescent field depth $d_{p}$ of $95 \mathrm{~nm}$. In this model it is assumed that backscattering of the evanescent wave and reflection of the scattered wave at the interface cause no significant perturbations (Prieve et al 1987), and that the optical properties of the particles are uniform. A relative change in particle height $\Delta h$ can be determined without knowing $I_{0}$ by taking the ratio in intensity between the two different heights $I_{1} / I_{2}$, and rewriting equation (1) to: 


$$
\Delta h=d_{p} \ln \frac{I_{1}}{I_{2}} .
$$

The height resolution is determined by the minimum change in intensity that can be measured. For a standard 8-bit CCD camera with 255 intensity levels, the minimum change in intensity is expected to be approximately $1 \%$, giving a height resolution of $1 \mathrm{~nm}$ in a field depth of $95 \mathrm{~nm}$. However, a change of $1^{\circ}$ in the angle of incidence of the exciting beam changes the evanescent field depth with $4 \%$, which directly relates to a fictional height change of $4 \%$. Depending on the local field depth, this can lead to errors up to $4 \mathrm{~nm}$ for height displacements in the order of $100 \mathrm{~nm}$. For $3^{\circ}$ angle variations the error amounts to $16 \mathrm{~nm}$. Thus, for nanometre accuracy it is important to have a collimated incident light beam with small variation in angle of incidence at the surface. We note that for optimal resolution in a biosensor system, the evanescent field depth may be tuned by changing the wavelength of the light $\lambda$ or the angle of incidence $\theta$; a lower evanescent field depth will give a higher resolution over a smaller range of interest. The minimum evanescent field depth is, however, limited by the refractive indices of the materials $(\sim 70 \mathrm{~nm}$ at $\lambda=650 \mathrm{~nm}$ or $\sim 40 \mathrm{~nm}$ at $\lambda=380 \mathrm{~nm}$ ).

Secondly, the average distance $\langle x\rangle$ travelled by a particle due to Brownian motion in a certain time interval $t$ is:

$$
<x>=2 \sqrt{D t / \pi} \text { with } D=k_{B} T / 6 \pi \eta r,
$$

where $D$ is the diffusion coefficient, $k_{B} T$ the thermal energy, $\eta$ the fluid viscosity and $r$ the particle radius. A particle with a diameter of $500 \mathrm{~nm}$ can travel in bulk fluid $30 \mathrm{~nm}$ in $1 \mathrm{~ms}$, or $1 \mathrm{~nm}$ in $1 \mu \mathrm{s}$. However, close to a surface the diffusion perpendicular to the surface is slower due to particle-wall interactions (Sholl et al 2000): $D^{*}=s_{\perp} D$, with:

$$
s_{\perp}=\frac{6 h^{2}+2 r h}{6 h^{2}+9 r h+2 r^{2}} .
$$

Taking for example a height of $30 \mathrm{~nm}$ gives $s_{\perp}=0.1$ and an average displacement of $10 \mathrm{~nm}$ in $1 \mathrm{~ms}$. Taking a height of $10 \mathrm{~nm}$ gives $s_{\perp}=0.04$ and an average displacement of $6 \mathrm{~nm}$ in $1 \mathrm{~ms}$. In addition, a particle that is biologically bound may also dissipate energy by molecular deformation (bending, stretching or rotation) slowing down its diffusion with an additional factor, whose value cannot easily be estimated.

In order to obtain nanometre height resolution by measuring the scattered light of a particle it is required that the duration of each intensity measurement is not much larger than the time it takes for the particle to move a nanometre. We estimate that the typical measurement time for $500 \mathrm{~nm}$ particles should be at maximum $1 \mathrm{~ms}$. Achieving this temporal resolution in a compact evanescent wave biosensor is challenging, because small light sources that can be integrated into a compact system have limited light power. The light power can be increased by focusing the light onto the sensor surface. However, the focusing angles should remain small, as described above, to assure that the evanescent field depth is constant and well-defined over the field of view of the sensor surface. In our evanescent wave biosensor, we have chosen to use a small diode laser combined with a lens, and non-fluorescent particle labels for optimum light efficiency. Finally, while the time per measurement limits the height resolution, the measurement frequency is not critical due to the statistical nature of the particle mobility. Therefore time gating can be applied, which can be done either in the illumination path or in the detection path. In earlier tethered particle motion experiments time-gated stroboscopic illumination was used (Blumberg et al 2005). In an integrated biosensor system, time-gated detection is preferred in order to achieve an optimal signal-to-noise ratio. Time-gated detection can be implemented using a relatively standard CCD camera with low frame rates and the possibility to define the exposure time per frame.

\section{Materials and methods}

\subsection{Detection}

Our evanescent wave biosensor set-up with scattered light imaging is sketched in figure $1(a)$. A light beam is coupled into a disposable, injection moulded, polystyrene cartridge $(12 \times 40 \mathrm{~mm})$ with an angle of incidence of $70^{\circ}$, using optical entrance facets perpendicular to the light beam (Bruls et al 2009). At the sensor area the 
cartridge thickness is $0.8 \mathrm{~mm}$. A $2 \mathrm{~mm}$ wide fluid channel is created with $180 \mu \mathrm{m}$ thick double sided tape and a $150 \mu \mathrm{m}$ thick microscope cover glass. As a light source we use a red laser diode (658 nm, Sanyo DL6147-240). The laser is slightly focused to an optical spot at the sensor area of approximately $200 \mu \mathrm{m}$ in diameter, with a maximum variation in the angle of incidence of $0.7^{\circ}$ with respect to the optical axis. The laser is operated at approximately $20 \mathrm{~mW}$, leading to a power density of approximately $6.10^{2} \mathrm{~mW} / \mathrm{mm}^{2}$ at the sensor surface, which is sufficient to measure with $1 \mathrm{~ms}$ camera exposure times. As a control light source, we also use a high power LED (620-645 nm, Luxeon LXHL-BD03), which is less sensitive to interference effects because of its lower coherence. The optical output of the LED is approximately $160 \mathrm{~mW}$. The light of the LED is quasi-collimated using a set of diaphragms guaranteeing a maximum divergence angle of $1^{\circ}$. As a result, the collection efficiency of the LED output is considerably reduced, and the measured optical power density at the sensor area is $0.08 \mathrm{~mW} / \mathrm{mm}^{2}$, resulting in camera exposure times in the order of seconds. The dimensions of the cartridge, cartridge holder and illumination system together are 20x10x6 cm.

(a)

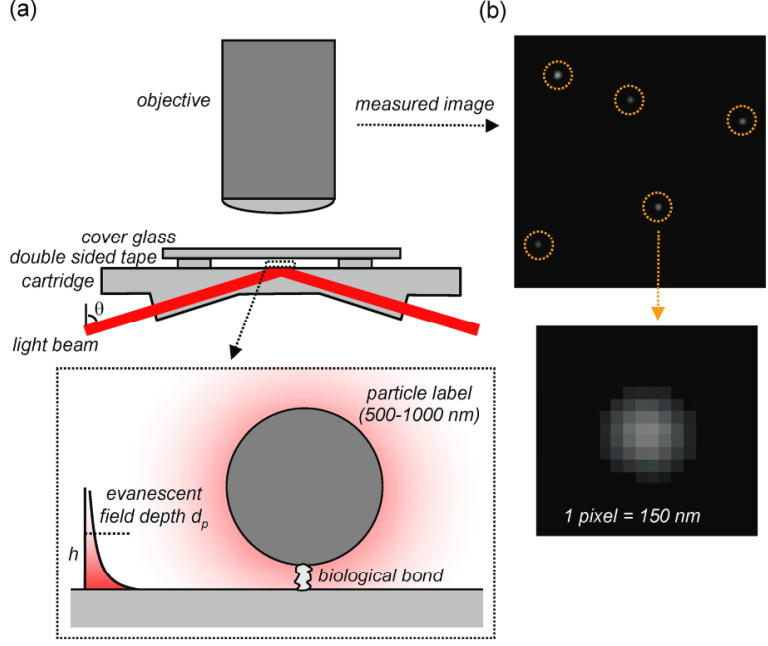

(c)

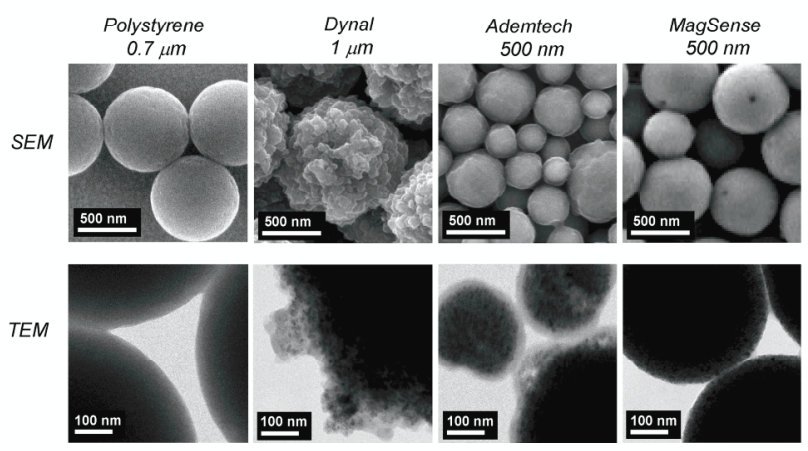

Figure 1. (a) Schematic representation of the optical evanescent wave biosensor set-up. The evanescent wave is formed by a light beam that reflects at the surface of an injection moulded cartridge under an angle $\theta$. The scattered light of biologically bound magnetic particles in the evanescent field is imaged with an objective lens and a camera. (b) Example of a measured image of particles in the evanescent field. (c) Scanning Electron Microscopy (SEM) and Tunnelling Electron Microscopy (TEM) images of the $0.7 \mu \mathrm{m}$ polystyrene particles and three types of magnetic particles: Dynal $1 \mu \mathrm{m}$, Ademtech $500 \mathrm{~nm}$ and MagSense $500 \mathrm{~nm}$.

The scattered light of the particles residing in the evanescent field is imaged with single particle resolution using a Leica microscope (DMLM/LP), a 50x objective lens with numerical aperture NA=0.50 (N plan) and a CCD camera (Pike, Allied Vision, minimum shutter speed $18 \mu \mathrm{s}$, maximum frame rate $120 \mathrm{~Hz}$ ). The field of view is $96 \mu \mathrm{m}$ by $72 \mu \mathrm{m}$ and one pixel corresponds to $150 \mathrm{~nm}$. An example of a measured particle image is shown in figure 1(b), and an example of a part of a recorded movie can be found in the Supplementary Material. To analyze the recorded movies, we developed video analysis software in Matlab that recognizes the particles, determines the $x-y$ position with sub-pixel accuracy $(\sim 15 \mathrm{~nm})$ by a centre of mass analysis of the intensity profile, and calculates the total measured intensity per particle by integrating all pixels belonging to a single particle.

As detection labels we use both polystyrene particles and different types of magnetic particles. The polystyrene particles are 0.7-0.9 $\mu \mathrm{m}$ polystyrene particles with a streptavidin coating of Spherotech Inc. The magnetic particles are $500 \mathrm{~nm}$ to $1000 \mathrm{~nm}$ superparamagnetic beads, consisting of small $(5-15 \mathrm{~nm})$ iron oxide grains in a polystyrene matrix from three different manufacturers: streptavidin coated Dynal MyOne beads with a diameter of $1 \mu \mathrm{m}$, streptavidin coated Ademtech Masterbeads with a diameter of $500 \mathrm{~nm}$, and streptavidin coated MagSense beads with a diameter of $500 \mathrm{~nm}$. All particles are known for their high volume susceptibilities (>2). In figure 1(c) Scanning Electron Microscopy (SEM) and Tunnelling Electron 
Microscopy (TEM) images of all particle types are shown. The polystyrene particles are uniform in diameter (mean diameter $760 \mathrm{~nm}$, coefficient of variation (CV) of 5\%). Also the Dynal particles have a uniform diameter (mean diameter $1.05 \mu \mathrm{m}, \mathrm{CV}$ of 3\%). The Ademtech particles are quite polydisperse (mean diameter $420 \mathrm{~nm}, \mathrm{CV}$ of 25\%), and also the MagSense particle are polydisperse (mean diameter $580 \mathrm{~nm}, \mathrm{CV}$ of $25 \%$ ). The polystyrene particles are spherical, the shape and roughness of the different magnetic particles varies.

\subsection{Model assay}

As a model analyte for binding particles to the surface we use a $290 \mathrm{bp}(99 \mathrm{~nm})$ double-stranded PCR amplification product of an antibiotic resistance gene of Salmonella, which is tagged with Texas red on one end and biotin on the other end. Amplification has been described by Koets et al (2009). The DNA is diluted in $100 \mathrm{mM}$ borate buffer $(\mathrm{pH} 8.8)$ containing $1 \%(\mathrm{w} / \mathrm{v})$ BSA and $0.05 \%(\mathrm{v} / \mathrm{v})$ Tween 20 . The streptavidin coated particles are washed, magnetically or in a centrifuge, and diluted in the same buffer. The sensor surface is coated with polyclonal antibodies against Texas red using an inkjet printer $(2 \mathrm{nl}$ drop of $150 \mu \mathrm{g}$ antibody/ml in PBS buffer). The spot diameter is approximately $200 \mu \mathrm{m}$. After printing, the cartridges are blocked in a PBS buffer containing $1 \%(\mathrm{w} / \mathrm{v})$ BSA, $10 \%(\mathrm{w} / \mathrm{v})$ sucrose and $0.1 \%(\mathrm{w} / \mathrm{v})$ sodium azide. Before use, the cartridges are washed with $100 \mathrm{mM}$ borate buffer $(\mathrm{pH} 8.8)$ containing $0.05 \%(\mathrm{v} / \mathrm{v})$ Tween 20.

An assay is performed by adding the particle solution to the DNA solution, and incubating for 1 minute (Koets et al 2009). The cartridge channel is then filled with $4 \mu \mathrm{l}$ fluid and incubated for 3 minutes. Finally, unbound particles are washed away using a pipette with $400 \mu 100 \mathrm{mM}$ borate buffer $(\mathrm{pH} 8.8)$ containing $0.05 \%(\mathrm{v} / \mathrm{v})$ Tween 20 . For polystyrene particles the particle concentration is $0.25 \%(\mathrm{w} / \mathrm{v})$ and the DNA concentration $5 \mathrm{pM}$. For Ademtech $500 \mathrm{~nm}$ particles the particle concentration is $0.1 \%(\mathrm{w} / \mathrm{v})$ and the DNA concentration $10 \mathrm{pM}$. For the Dynal $1 \mu \mathrm{m}$ particles the particle concentration is $0.03 \%(\mathrm{w} / \mathrm{v})$ and the DNA concentration $0.3 \mathrm{pM}$. For the MagSense $500 \mathrm{~nm}$ particles the particle concentration is $0.1 \%(\mathrm{w} / \mathrm{v})$ and the DNA concentration $10 \mathrm{pM}$. For all particle types the particle concentration was experimentally tuned to obtain a similarly low density of bound particles on the sensor surface, and the DNA concentration was calculated to give slightly fewer DNA molecules than particles in the fluid, thus making double bonds between particles labels and surface unlikely.

\section{Results and discussion}

\subsection{Characterization of the set-up using fixated particles}

To determine the height resolution of our set-up, we analyzed the intensity fluctuations of particles that were fixated to the sensor surface. Fixating particles to the surface was done by first binding particles to the sensor surface, then drying the cartridge, and finally refilling the cartridge with water for the measurements. This preparation procedure led to immobile particles on the sensor surface.

In a first version of the optical system with laser illumination, measurements on fixated particles showed large changes in intensity of two distinct types: slow oscillations on the order of seconds, or sudden steps that appeared every few minutes. The occurrence of both phenomena appeared to be random, and the change in intensity occasionally was as large as $50 \%$. The intensity fluctuations were always synchronized for all particles in one experiment and often out of phase for different particles in the same image: one particle would become darker as the other would become lighter, which occurred randomly with respect to the position in the image. This effect was seen for all particle types (polystyrene and magnetic), but was absent when laser illumination was replaced by LED illumination. It is generally known that lasers in microscopy applications may generate intensity fluctuations (due to laser mode hopping) and interference effects (due to coherence of the light), limiting the sensitivity and reproducibility of the experiments (Pawley 1995). We minimized mechanical vibrations in the system by using a stable optical bench and we reduced the laser mode hopping noise by using RF modulation of the laser diode (Petermann 1988). These measures effectively removed both the oscillations and the sudden steps.

Figure 2(a) shows the intensity versus time traces for seven single fixated particles, recorded in the system with laser with the above mentioned modifications. The frame rate was $7.5 \mathrm{~Hz}$ and the exposure time $0.5 \mathrm{~ms}$. In the signals we observed small rapid fluctuations with a standard deviation lower than $1 \%$ and an additional slow drift with an amplitude of up to $6 \%$. We attribute the rapid fluctuations to the laser relative intensity noise (RIN). The slow drift signal was seen to either increase or decrease in repeated measurements 
and was not related to the measurement phase. To explain the drift we analyzed also the $x, y$-position data. Figure 2(b) shows that the system has a constant position drift of about $1 \mathrm{~nm} / \mathrm{second}$, but the position drift is not directly related to the intensity change. We further investigated the influence of particle position on the intensity by changing the position of the table (and thus the imaged particles) with respect to the imaging objective. We found that the system was mostly insensitive to $x, y$-displacements of a few pixels, but occasionally small displacements ( 0.5 pixels) lead to intensity changes up to $10 \%$. The occasional larger changes may be due to optical interference effects, which can generate sharp transitions in light intensity at the sensor surface. Another possibility is heating in the cartridge, leading to small changes in the light path, which can lead to variable interferences at the sensor surface.

(a)

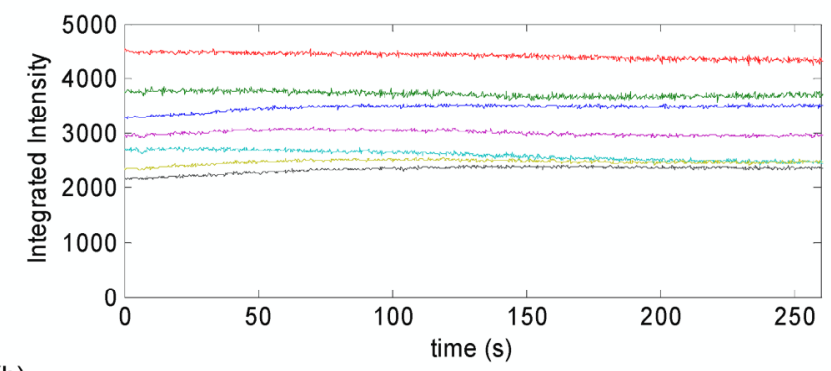

(b)
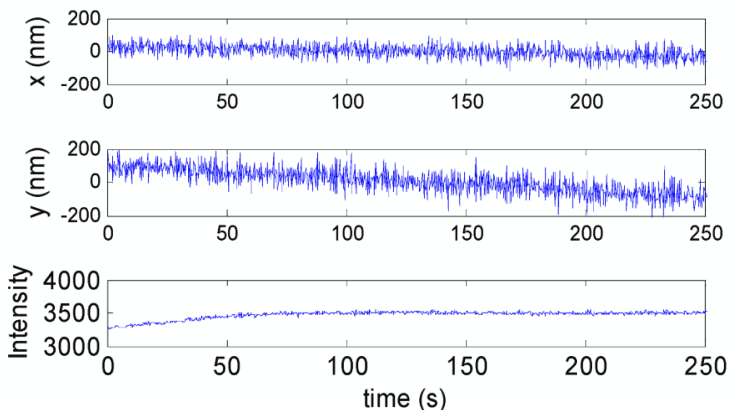

Figure 2. (a) Scattered light intensity versus time for seven magnetic (Ademtech $500 \mathrm{~nm}$ ) particles that were rigidly fixed to the surface showing fluctuations of less than $1 \%$ in magnitude and a slow drift in intensity of at maximum $6 \%$. (b) $x$-position, $y$-position and intensity as function of time, for one of the particles from graph $a$.

The maximum height resolution of our system can be determined from the measured intensity fluctuations due to noise and drift. The measured standard deviation due to noise of $1 \%$ gives a possible height resolution (see equation (3)) of $1 \mathrm{~nm}$. The intensity fluctuation due to drift is at maximum $10 \%$, giving a height resolution of $9 \mathrm{~nm}$. It should however be noted that in stable regions and for characteristic measurement times of around 30 seconds, errors due to drift could usually be neglected, so in our biosensor we are able to determine particle height with a resolution close to 1 nanometre.

Finally, we noticed that for the magnetic particles, the particle to particle difference in scattered light intensity within one type of particles was quite large. For both Ademtech and MagSense particles we observed more than a factor of 10 difference between particles, which might be explained by their wide size distribution (200-600 nm). However, also for Dynal particles we observed differences by a factor of 6, while these particles do have a very narrow size distribution $(\mathrm{CV}<3 \%)$. The large signal differences were also observed with LED illumination. These observations indicate that particle shape, particle roughness and magnetic content variations can be very important in optical scattering measurements.

\subsection{Intensity fluctuations of bound particles}

We measured the Brownian motion induced intensity fluctuations over time for the different particle types bound to the surface with $290 \mathrm{bp}(99 \mathrm{~nm})$ DNA (see movie example in the Supplementary Material). The $\mathrm{CCD}$ was operated at $60 \mathrm{~Hz}$ with an exposure time of $4 \mathrm{~ms}$ for polystyrene particles and between 0.4 and 1 $\mathrm{ms}$ for the magnetic particles. We analyzed the intensity data by making histograms and determining the 
minimum and maximum intensity. Typical histograms of a polystyrene particle and of the different magnetic particles are shown in figure $3(a)$.

(a) Intensity histograms
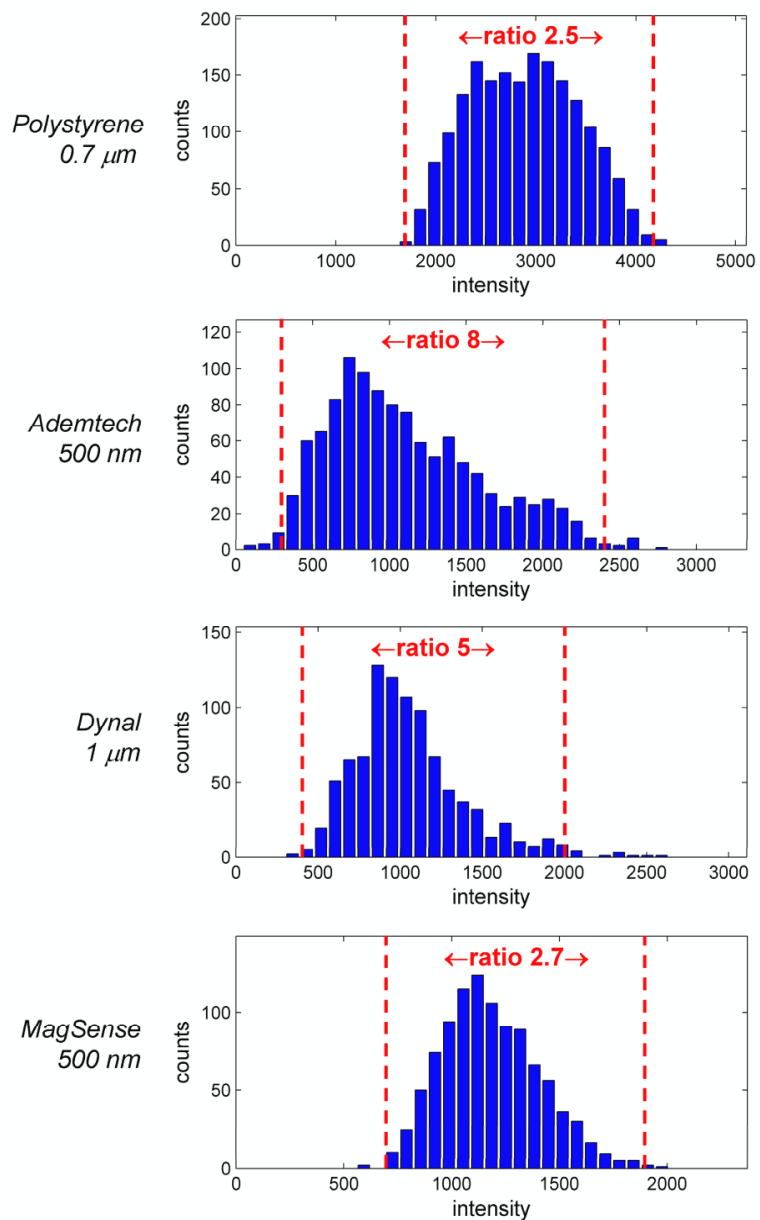

(b)

2D-position histograms

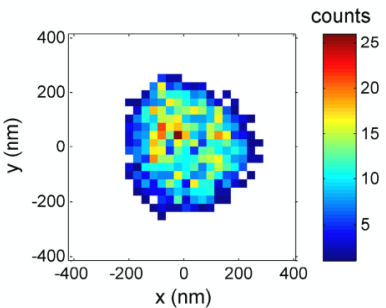

counts
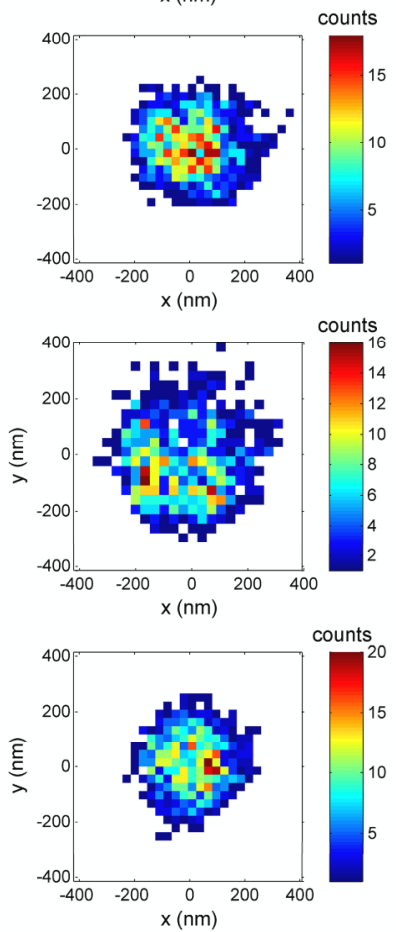

(c) Average intensity color plots
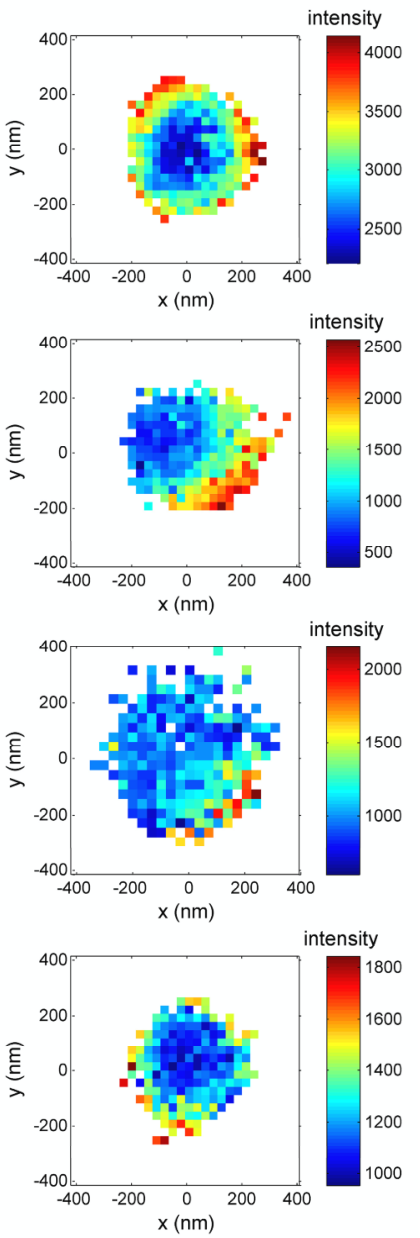

Figure 3. (a) Histograms of measured intensity values for a polystyrene $0.7 \mu \mathrm{m}$ particle, an Ademtech 500 $\mathrm{nm}$ particle, a Dynal $1 \mu \mathrm{m}$ particle and a MagSense $500 \mathrm{~nm}$ particle, bound to $99 \mathrm{~nm}$ DNA in a $95 \mathrm{~nm}$ evanescent field, measured in 30 seconds (60 fps). (b) Colour plots of the two-dimensional histograms of the $x$ - and $y$-position data of the same particles as in $a$. (c) Colour plots of the average intensity found at each position for the same particles as in $a$ and $b$.

The histogram of the polystyrene particle in figure 3(a) shows an intensity ratio of 2.5 . For other polystyrene particles we observed ratios mostly below 3. For an estimated evanescent field depth in our system of $95 \mathrm{~nm}$, and a maximum height displacement of $99 \mathrm{~nm}$ (the DNA length), we calculate a maximum intensity ratio of about 2.8 using the ideal exponential decay model (equation (1)). Adding the size of the antibodies and linker molecules (total length approximately $20 \mathrm{~nm}$ ) gives a maximum possible intensity ratio of about 3.5. The intensity ratios found in the experiments are thus consistent with the expected bond length. It should be noted that we also observed lower ratios down to 1 , which we attribute to different binding types, such as additional non-specific sticking of specifically bound beads, which we will further explore in future work. Finally, the measured values for the polystyrene particles may be underestimated due to the relatively high exposure times for these particles $(4 \mathrm{~ms})$, which is slightly larger than we estimate to be advisable for $760 \mathrm{~nm}$ particles $(<2 \mathrm{~ms})$. The slightly longer exposure time could lead to an error in detecting the extreme positions (on the order of a few nanometres) and therefore to slightly lower measured intensity ratios (on the order of $3 \%$ ).

The histogram for the Ademtech $500 \mathrm{~nm}$ particle in figure 3(a) shows a ratio of a factor 8, which is much larger than the calculated maximum ratio. A factor 8 corresponds to a height change of $198 \mathrm{~nm}$, which 
clearly exceeds the length of the bond. Also, the Dynal $1 \mu \mathrm{m}$ particle in figure 3(a) shows a large ratio of 5 . For other Ademtech and Dynal particles we measured ratios between 3 and 10. Finally, the histogram of the MagSense $500 \mathrm{~nm}$ particle shows a ratio of 2.7, which is again below the maximum calculated intensity ratio. For other MagSense particle we observed ratio's mostly below 3.5. Intensity ratios that are higher than expected from the maximum bond height displacement, might be explained by a deviation from the idealized exponential decay model. Recently, it has been shown that multiple reflections between a particle and the surface can cause a distortion of the exponential model, especially for large penetration depths (close to half the excitation wavelength) and small particle heights (Helden et al 2006, Hertlein et al 2008). For a small penetration depth of $132 \mathrm{~nm}$, which is on the order of the $95 \mathrm{~nm}$ penetration depth in our system, simulations with $1.6 \mu \mathrm{m}$ diameter polystyrene particles pointed to a reduction in scattered light intensity near the surface of approximately $15 \%$, leading to a reduced measured height change. This effect is small compared to our observations and also opposes the result of measuring higher intensity ratios than expected.

Another way to analyze the mobility data measured in an evanescent wave biosensor is to combine the intensity information with the in-plane position data. First, we studied the in-plane positions $(x, y)$ of the imaged particles by making colour plots of the two-dimensional positional histograms. Figure $3(b)$ shows these histograms for the particles of figure 3(a). All particles appear to move in a circle with a radius of 200$300 \mathrm{~nm}$. Geometrically, the maximum radius of the circle can be estimated by $x=\sqrt{l_{D N A}{ }^{2}+2 r l_{D N A}}$ with $l_{D N A}$ the DNA length. For a particle with a diameter of $900 \mathrm{~nm}$ and a DNA length of $99 \mathrm{~nm}$, the maximum radius is $322 \mathrm{~nm}$. For a $500 \mathrm{~nm}$ particle, the maximum radius is $244 \mathrm{~nm}$. Thus, the measured radii are indeed in the expected range. Moreover, for all particles we observe a maximum of the position histograms near the middle of the position scatter plots. One might hypothesize that the centred histogram is caused by electrostatic repulsion between particle and surface. However, estimations using the approach of Leckband and Israelachvili (2001) show that in our buffer conditions electrostatic repulsion can be neglected for particle heights larger than $6 \mathrm{~nm}$. A probable cause for the centred histograms is a preferred upright orientation of the analyte-antibody complex combined with a flexibility of the analyte molecule. dsDNA is known to be a semiflexible molecule with a persistence length of around $50 \mathrm{~nm}$ (Brinkers et al 2009, Manning 2006). A flexible analyte has a higher density of states at the centre position, and thus a higher probability to be at that position.

To further study the analyte properties, we determined the average intensity over time at each measured $x, y$-position. Figure 3(c) shows the colour plots of the average intensity for the particles of figure $3(a)$ and $3(b)$. For a tethered particle, the lowest values for the average intensity (i.e. largest height) are expected to occur in the centre of the circle and the highest intensity values (i.e. lowest height) are expected to occur at the contour of the circle, because the analyte-antibody complex has to tilt or bend in order for the particle to touch the surface. For the polystyrene particle the abovementioned intensity-position relationship is indeed observed, see figure $3(\mathrm{c})$. Moreover, the average intensity at the centre position is about $20 \%$ lower than the lowest value in the intensity histogram, which shows that the analyte indeed has flexibility. A simple model for the density of states shows that this flexibility is sufficient to account for the centred maximum in the position histogram. Additional thermodynamic modelling will allow the extraction of more detailed analyte information from the data.

Also for the MagSense magnetic particle, which showed the expected maximum intensity ratio, a relatively symmetric intensity plot can be seen with the lowest values at the centre and the highest values at the contour of the circle. Most other polystyrene and MagSense particles showed similar intensity profiles. However, for both the Ademtech magnetic particle and the Dynal magnetic particle a completely different result is seen. Although the position histogram for the Ademtech particle in figure 3(b) shows a preference for the middle position, the highest intensity values in figure 3(c) are obtained in only one direction. Combined with the fact that the fluctuations are much larger than expected, these data suggest that nonuniform particle properties combined with rotational freedom of the particle are of large influence, leading to intensity changes that dominate over height dependence, with the consequence that equation (1) can not be applied. Also the Dynal particle depicted in figure 3(c) shows an asymmetric intensity colour plot. For different Ademtech and Dynal particles a variety of intensity profiles could be found.

In the SEM and TEM pictures of figure 1(c) it can be seen that both Dynal particles and Ademtech particles have a considerable surface roughness. Ademtech particles are observed to have a well-defined polystyrene shell of $25 \mathrm{~nm}$, but often have a few protrusions up to $60 \mathrm{~nm}$ in size, that can sometimes differ in 
magnetic content. Moreover, shape aspect ratios up to 1.2 are not uncommon and inhomogeneous magnetic content can sometimes be seen. Dynal particles have a less well-defined polystyrene shell of $25 \mathrm{~nm}$ containing still some iron oxide grains. All Dynal particles have many protrusions up to $100 \mathrm{~nm}$ in size, and these parts can sometimes vary in magnetic content. These observations are likely to explain the large differences in signal observed in an evanescent field of $95 \mathrm{~nm}$, due to the fact that bound particles will also have the freedom to not only change height, but also to tilt and rotate. The MagSense particles are much smoother with no visible shell, a surface roughness of less than $10 \mathrm{~nm}$, and no inhomogeneous iron oxide content. Therefore, it can indeed be expected that these particles behave more uniformly in the evanescent field. The only irregularity in MagSense particles is the occurrence of a small pit, usually one per particle, with an estimated maximum diameter of $50 \mathrm{~nm}$, which may still influence the optical signal when the pit faces the sensor surface. One could limit the freedom of rotation of magnetic particles, and therefore the influence of non-uniform optical properties on the intensity signal, by application of a magnetic field (Blickle et al 2005). However, for bound particles an inhibition of particle rotation limits the height mobility of the particles and thereby decreases the molecular information that can be derived from the optical signals.

The results demonstrate that the height mobility of biologically bound polystyrene and magnetic particles can be determined by measuring the intensity fluctuations of particle-induced light scattering. The polystyrene particles and the MagSense particles are highly uniform, so allow quantitative detection of height changes. The Ademtech and Dynal magnetic particles have non-uniform optical properties. The mobility of such particles generates high intensity fluctuations that have an indirect relationship with the particle height, so these particles are suited for qualitative height mobility detection.

\section{Conclusions}

We have built a compact biosensor set-up to detect individual particle labels and quantify their thermally induced mobility on the biosensor surface with nanometre resolution. Surface and height sensitive detection was made possible by using an evanescent optical field and subsequent imaging of the scattered light from the particles. We performed assays with 500 to $1000 \mathrm{~nm}$ particle labels that were bound to a sensor surface with $290 \mathrm{bp}(99 \mathrm{~nm}) \mathrm{dsDNA}$ as a model analyte.

For polystyrene particles the maximum intensity variations are about a factor of 3 , which is close to the calculated value of 2.8 for $99 \mathrm{~nm}$ DNA in a $95 \mathrm{~nm}$ evanescent field. Further analysis of the $x, y$-position data shows that the positions are distributed with circular symmetry. The particles have a preference for a centered position where the average height is highest and show the lowest heights at the contour of the circle, which is a reflection of the analyte-antibody orientation and the analyte flexibility. For the smooth MagSense magnetic particles (surface roughness $<10 \mathrm{~nm}$ ), the maximum intensity ratio is about a factor of 3.5, which corresponds to a calculated height displacement of $120 \mathrm{~nm}$. The slightly larger height can be due to the additional antibody and linker molecules. For both Ademtech and Dynal magnetic particles, the maximum intensity ratio goes up to a factor of 10 , which we attribute to particle properties such as non-spherical shape, surface roughness and inhomogeneous magnetic content. Ademtech and Dynal magnetic particles are thus only suitable for qualitative mobility detection.

Summarizing, the evanescent-wave excitation and imaging technique gives single-particle resolution and precise intensity information, and therefore gives an accurate characterization of the mobility of particle labels that are biologically bound to a substrate. A next step is to investigate the novel optical biosensor technique for a range of different biological analytes. We believe that this technique will pave the way for biosensing with very high sensitivity and specificity.

\section{Acknowledgment}

This work was partially funded by the NanoNed program of the Dutch Ministry of Economic Affairs.

\section{References}

Banerjee A and Kihm K D 2005 Experimental verification of near-wall hindered diffusion for the Brownian motion of nanoparticles using evanescent wave microscopy Phys. Rev. E 72042101

Blickle V, Babič D and Bechinger C 2005 Evanescent light scattering with magnetic colloids Appl. Phys. Lett. 87101102

Blumberg S, Gajraj A, Pennington M W and Meiners J-C 2005 Three-dimensional characterization of tethered microspheres by total internal reflection fluorescence microscopy Biophys. J. 89 1272-81 
Brinkers S, Dietrich H R C, de Groote F H, Young I T and Rieger B 2009 The persistence length of double stranded DNA determined using dark field tethered particle motion J. Chem. Phys. 130215105

Bruls D M et al 2009 Rapid integrated biosensor for multiplexed immunoassays based on actuated magnetic nanoparticles Lab Chip 93504

Graham D L, Ferreira H A and Freitas P P 2004 Magnetoresistive-based biosensors and biochips Trends in Biotech. 22 455-62

Helden L, Eremina E, Riefler N, Hertlein C, Bechinger C, Eremin Y and Wriedt T 2006 Single-particle evanescent light scattering simulations for total internal reflection microscopy Appl. Opt. 45 7299308

Hertlein C, Riefler N, Eremina E, Wriedt T, Eremin Y, Helden L and Bechinger C 2008 Experimental verification of an exact evanescent light scattering model for TIRM Langmuir 24 1-4

Koets M, van der Wijk T, van Eemeren J T W M, van Amerongen A and Prins M W J 2009 Rapid DNA multi-analyte immunoassay on a magneto-resistance biosensor Biosens. Bioelectron. 24 1893-8

Leckband D and Israelachvili J 2001 Intermolecular forces in biology Q. Rev. Biophys. 34 105-267

Manning G S 2006 The persistence length of DNA is reached from the persistence length of its null isomer through an internal electrostatic stretching force Biophys. J. 91 3607-16

Mulvaney S P, Myers K M, Sheehan P E and Whitman L J 2009 Attomolar protein detection in complex sample matrices with semi-homogeneous fluidic force discrimination assays Biosens. Bioelectron. 24 1109-15

Oetama R J and Walz J Y 2005 A new approach for analyzing particle motion near an interface using total internal reflection microscopy J. Coll. Int. Sci. 284 323-31

Osterfeld S J et al 2008 Multiplex protein assays based on real-time magnetic nanotag sensing PNAS 105 20637-40

Pawley J B 1995 Handbook of biological confocal microscopy (New York: Springer Science+Business Media, Inc.)

Petermann K 1988 Laser Diode Modulation and Noise (Dordrecht: Kluwer Academic Publishers)

Prieve D C 1999 Measurement of colloidal forces with TIRM Adv. Coll. Int. Sci. 82 93-125

Prieve D C, Luo F and Lanni F 1987 Brownian motion of a hydrosol particle in a colloidal force field Faraday Discuss. Chem. Soc. 83 297-307

Seydack M 2005 Nanoparticle labels in immunosensing using optical detection methods Biosens. Bioelectron. $202454-69$

Sholl D S, Fenwick M K, Atman E and Prieve D C 2000 Brownian dynamics simulation of the motion of a rigid sphere in a viscous fluid very near a wall J. Chem. Phys. 113 9268-78

Wild D 2005 The Immunoassay Handbook (Amsterdam: Elsevier) 